\title{
Frontal midline theta and the error-related negativity: neurophysiological mechanisms of action regulation
}

\author{
Phan Luu ${ }^{\mathrm{a}, \mathrm{b}, *}$, Don M. Tucker ${ }^{\mathrm{a}, \mathrm{b}}$, Scott Makeig ${ }^{\mathrm{c}}$ \\ ${ }^{a}$ Electrical Geodesics, Inc., 1600 Millrace Dr Suite 307, Eugene, OR 97403, USA \\ ${ }^{\mathrm{b}}$ Department of Psychology, University of Oregon, Eugene, OR 97403, USA \\ ${ }^{\mathrm{c}}$ Swartz Center for Computational Neuroscience, Institute for Neural Computation, University of California San Diego, La Jolla, CA 92093-0961, USA
}

Accepted 22 March 2004

Available online 20 May 2004

\begin{abstract}
Objective: The error-related negativity (ERN) is an event-related potential (ERP) peak occurring between 50 and $100 \mathrm{~ms}$ after the commission of a speeded motor response that the subject immediately realizes to be in error. The ERN is believed to index brain processes that monitor action outcomes. Our previous analyses of ERP and EEG data suggested that the ERN is dominated by partial phase-locking of intermittent theta-band EEG activity. In this paper, this possibility is further evaluated.

Methods: The possibility that the ERN is produced by phase-locking of theta-band EEG activity was examined by analyzing the singletrial EEG traces from a forced-choice speeded response paradigm before and after applying theta-band (4-7 Hz) filtering and by comparing the averaged and single-trial phase-locked (ERP) and non-phase-locked (other) EEG data. Electrical source analyses were used to estimate the brain sources involved in the generation of the ERN.

Results: Beginning just before incorrect button presses in a speeded choice response paradigm, midfrontal theta-band activity increased in amplitude and became partially and transiently phase-locked to the subject's motor response, accounting for 57\% of ERN peak amplitude. The portion of the theta-EEG activity increase remaining after subtracting the response-locked ERP from each trial was larger and longer lasting after error responses than after correct responses, extending on average $400 \mathrm{~ms}$ beyond the ERN peak. Multiple equivalent-dipole source analysis suggested 3 possible equivalent dipole sources of the theta-bandpassed ERN, while the scalp distribution of non-phase-locked theta amplitude suggested the presence of additional frontal theta-EEG sources.

Conclusions: These results appear consistent with a body of research that demonstrates a relationship between limbic theta activity and action regulation, including error monitoring and learning.
\end{abstract}

(C) 2004 International Federation of Clinical Neurophysiology. Published by Elsevier Ireland Ltd. All rights reserved.

Keywords: Anterior cingulate cortex; Supplementary motor area; Error-related negativity; Motor potentials; Event-related potentials; Theta; Oscillations; Phase resetting

\section{Introduction}

Self-monitoring of the consequences of action is an important self-regulatory function. The identification of the error-related negativity (ERN or Ne) by Falkenstein et al. (1991) and Gehring et al. (1993) has proved to be one of the more exciting discoveries in psychophysiological research in the past decade. Over 50 studies of the ERN have been

\footnotetext{
* Corresponding author. Address: Department of Psychology, University of Oregon, Eugene, OR 97403, USA. Tel.: +541-687-7962; fax: +541687-7963.

E-mail addresses: pluu@oregon.uoregon.edu (P. Luu); dtucker@ oregon.uoregon.edu (D.M. Tucker); smakeig@ucsd.edu (S. Makeig).
}

published, and a number of fMRI studies have been inspired by the ERN literature (Carter et al., 1998). However, the significance of this signal remains controversial.

The tentative localization of the ERN generator to within or near dorsal anterior cingulate cortex (ACC) (Dehaene et al., 1994) was consistent with fMRI and positron emission tomography literature implicating the ACC in executive control. Subsequently, it was suggested that the ERN is not an index of error monitoring per se, but rather an index of response conflict monitoring in the ACC (Carter et al., 1998). There is supporting evidence for both the error monitoring and conflict monitoring views (see Luu and Tucker, 2003). An alternative and more general view is that the ERN may index affective evaluation, of errors in 
particular and of action outcomes in general (Luu et al., 2003; Vidal et al., 2000).

Less well known is evidence that the ERN may arise in large part from error-induced phase consistency of ongoing midline frontal theta $(4-7 \mathrm{~Hz})$ EEG activity (Luu and Tucker, 2001; Makeig et al., 2002). A clue to this possibility was the observation that the ERN may be both preceded and followed by positive and negative peaks in the ERP waveform at approximately $200 \mathrm{~ms}$ intervals. Luu and Tucker (2001) filtered the ERPs from a speeded response task within a 4-12 Hz bandpass. The ERN at frontal midline sites then appeared to be part of a 2-3 cycle midline oscillatory phenomenon. In error trials, the ERN was accompanied by theta-band EEG activity recorded above motor cortex. Preliminary independent component analysis (ICA) by Makeig et al. (2002) of the same data revealed that the largest independent EEG component contributors to the ERN produced oscillatory activity in the theta-frequency range. In another study, a midline frontal theta-source model inspired by the magnetoencephalography (MEG) study of Asada and colleagues (1999) was shown to account for the ERN at medial frontal scalp sites (Luu et al., 2003).

Although the possible relationship between the ERN and limbic theta rhythms has not been discussed in the recent ERN literature, such a relationship was anticipated by findings by Gevins and colleagues over a decade ago. Gevins et al. (1989) found that when subjects were provided with an error feedback signal, theta-band activity over midline frontal sites became correlated with theta-band activity over left lateral frontal sites. This pattern of results was not observed following feedback for correct performance. This difference emerged approximately $280 \mathrm{~ms}$ after onset of the feedback stimulus. We have observed a similar midline frontal theta response in the same time period, when subjects are provided with feedback about their performance. It is possible that this effect is intimately related to the appearance of the ERN (Luu et al., 2003).

The recognition that ERP components may result from relative phase consistency, with respect to stimulus delivery, of ongoing EEG rhythms is not new. In 1974, Sayers et al. (1974) noted that auditory ERPs could be evoked by stimuli that produced no increase in EEG power. Basar and colleagues $(1998,1992)$ have also proposed for many years that ERP components can reflect the summing up of phase-aligned oscillatory EEG activity. Recently, Makeig et al. (2002b) demonstrated how familiar visual ERP features, in particular 'alpha ringing' as well as portions of the visual N1, could arise from induced statistical post-stimulus phase imbalance in ongoing alpha and theta rhythms sometimes termed partial phase locking or phase resetting. In that study, the same spatial sources were identified by ICA as contributing theta- and alpha-band features to both the averaged ERP and the ongoing pre-stimulus EEG. In particular, Makeig et al. (see their Fig. 4) concluded that transient and partial 'theta ringing' of midfrontal theta-EEG sources contributed strongly to the early visual N1 potential recorded over frontal scalp sites.
However, many researchers who study averaged ERPs may remain unconvinced by applications of frequencydomain methods to characterize dynamics of evoked responses. As is well-known, bandpass filtering may introduce artifacts including ringing from non-oscillatory transients. Although signal processing methods can quantify oscillatory features, visual inspection of single-trial traces may also be useful for studying the signal context in which the ERN emerges.

In this report, we extend the findings of Luu and Tucker (2001) and the preliminary report of Makeig et al. (2002) by demonstrating, first by visual inspection of unaveraged single-trial records, that the ERN occurs during partially phase-aligned bursts of theta waves in the ongoing EEG. Next, inspection of mean theta-band activity in individual trials confirms that error responses are associated with a period of increased theta activity just prior to and following the behavioral response. Because much of this activity is not reliably phase-locked to the motor response, it is not visible in the response-locked ERP.

We separately assess the phase-locked and non-phaselocked EEG data associated with correct and error responses, and show that the ERN may well result in large part from summation of brief, partially phase-locked post-response theta bursts, whose mean amplitude increase is larger and longer-lasting following error responses. We note results of another experiment showing that brief theta bursts dominate the frontal midline response during speeded selective responding. Finally, we derive a multi-dipole model of the ERN based on assumptions derived from previous evidence and fit to the grand-average response data. While the model accounts for much of the ERN waveform, it cannot account for the full distribution of theta energy in the EEG following the response. We discuss, therefore, the need for more complete examination of the event-related EEG dynamics accompanying and underlying the ERN.

\section{Methods}

\subsection{Participants}

Eleven participants were recruited from the University of Oregon Psychology Department subject pool (9 males, two females). Seven participants were right handed, the other 4 left handed. The mean age of the group was $19(\mathrm{SD}=1.04)$. Participants gave informed consent and participated in the study as partial fulfillment of course requirements. All participants had normal or corrected-to-normal vision and none reported taking medications or drugs. Prior to application of the Geodesic Sensor Net (Electrical Geodesics, Inc.), participants filled out several brief mood questionnaires. After participants were prepared for EEG recording, they were seated $60 \mathrm{~cm}$ in front of a computer monitor; a chin rest controlled distance and alignment and minimized head movements. To motivate participants to 
perform well, they were informed that an 'A' feedback letter, awarded for quick, correct responses, would be worth 4 points, ' $C$ ' feedback, awarded for average-latency correct responses, would be worth two points, that a late-response ' $F$ ' earned no points, and an ' $E$ ' (error) would result in a loss of two points. At the end of the study, subjects were paid a monetary bonus proportional to their accumulated point total.

\subsection{Task}

The task employed in this study is similar to methods developed by Derryberry to provide emotional feedback not directly related to response correction (Derryberry, 1990, 1991; Luu et al., 2003). In each trial, the performance feedback reflected subject performance on the 5 th previous trial. Each trial (number $N)$ thus began with a $\left(2 \times 1.5 \mathrm{~cm}^{2}\right)$ feedback signal (a letter A, C, F, or E) replacing the fixation square. In the first 5 trials of each block, an (meaningless) $\mathrm{X}$ was presented. Thereafter, the feedback letter reflected performance on trial $N-5$. The subjects were informed of the delayed nature of the feedback, whose purpose was to minimize the degree to which expectancy and performance in one trial would influence performance on the next trial. Five hundred milliseconds after feedback onset, a white target arrow $\left(1.3 \times 1.6 \mathrm{~cm}^{2}\right)$ appeared $8 \mathrm{~cm}$ to the left or right of the feedback letter.

Participants were instructed to respond to the target arrow by pressing a button with the hand indicated by the direction in which the arrow pointed. Arrows pointing in the same direction as the visual field (left or right of feedback) were termed to constitute compatible response demands, while arrows pointing in the direction opposite to the visual field in which they appeared constituted incompatible response demands. For example, a correct incompatible response was a button press with the index finger of the left hand in response to a leftward pointing arrow appearing to the right of the feedback, and an error was a response made with the right index finger. The number of compatible and incompatible response trials were equal. Immediately after the response, the feedback and target stimuli were replaced with the fixation square. Intertrial interval varied randomly between 1.5 and $2.5 \mathrm{~s}$.

The study consisted of 800 trials presented in 8100 -trial blocks. Four hundred trials used an 'Easy' cutoff responsetime criterion to determine the letter-grade feedback, whereas the other 400 trials used a 'Difficult' cutoff response-time criterion. The order of these criteria conditions was counterbalanced across subjects. The feedback letter for each trial was determined by comparing subject reaction time (RT) on each trial to criteria derived from the RT distributions of compatible and incompatible responses, respectively, during the previous block of 100 trials. In the Easy cutoff condition, correct responses quicker than the 34th percentile of each distribution received an 'A,' those slower than the 66th percentile received an ' $F$,' and those falling between these thresholds received a 'C.' The Difficult cutoff condition divided the RT distribution into 3 similar but unequally distributed percentile bands $(25,25$ and $50 \%$ ). The cutoff criterion used during the first 100-trial block was determined from a set of practice blocks in which subjects were presented with separate blocks of 40 compatible and 40 incompatible trials. The first 10 trials from each practice block were used to orient participants to the task and were not included in determining the initial cutoff criteria.

\subsection{EEG Recording}

EEG was recorded from 128 scalp sites using the Geodesic Sensor Net (Tucker, 1993). All electrode impedances were brought below $50 \mathrm{~K} \Omega$ before recording. All channels were referenced to $\mathrm{Cz}$ and collected using a $0.1-50 \mathrm{~Hz}$ bandpass. Signals were sampled at 125 samples/ $\mathrm{s}$ and digitized with a 12 bit $\mathrm{A} / \mathrm{D}$ converter.

\subsection{ERP and EEG Analysis}

The continuous EEG data were filtered with a $4-7 \mathrm{~Hz}$ bandpass using a finite impulse response (FIR) filter with zero phase distortion. The filter was set with a pass band attenuation of $0.1 \mathrm{~dB}$ and stop band attenuation of $40 \mathrm{~dB}$ with a $4 \mathrm{~Hz}$ transition band. Trials time-locked to correct and error responses were then extracted from the wide-band $(0.1-30 \mathrm{~Hz})$ and theta-band $(4-7 \mathrm{~Hz})$ filtered data. At this stage, all trials were corrected for ocular artifacts using the method of Gratton et al. (1983). After removal of ocular artifacts, the data were analyzed for additional artifacts. For theta-band data, trials with 10 or more channels recording voltage deviations exceeding $50 \mu \mathrm{V}$ (absolute) or $25 \mu \mathrm{V}$ (sample-to-sample difference) were excluded from the average. On those trials with fewer than 10 such channels, the 'bad-channel' data were replaced using spherical spline interpolation of neighboring channel values (Perrin et al., 1987). Prior to averaging the data, each trial was rereferenced to the average of all of the sensors at each time point. Finally, the correct and error trial subsets were each averaged.

Mean theta activity both time locked and phase locked to the responses were assessed in the resulting response epochs by response averaging. To determine the non-phase-locked theta-band activity, the theta-bandpassed ERP for each subject was first subtracted from each of the subject's thetabandpassed EEG data trials. The remaining single-trial data were then rectified prior to averaging to assess the mean time course of non-phase-locked theta amplitude across the trials.

\subsection{ERP source analysis}

Source analysis of the generators of the theta-band ERPs was based on results of previous analysis that suggested the ERN is related to one or more a frontal midline theta (fMT) 
processes (Luu and Tucker, 2001; Luu et al., 2003). Asada et al. (1999) found that two midline MEG sources coherently active in the theta band with a phase difference were required to account for the appearance of fMT during mental arithmetic. The first two authors tested this model on another ERN EEG data set and found that, indeed, two theta dipole sources approximately $50^{\circ}$ out of phase could also account for the theta-bandpassed ERN (Luu et al., 2003).

Here, source analysis was performed using BESA 2000 software (MEGIS, 2000), which localizes equivalent dipole sources in a 4-shell, ellipsoidal head model. We have recently reported finding possible bilateral insular sources of an ERP component related to the ERN in 256-channel EEG recordings (Tucker et al., 2004). The present data were collected with a 128-channel array that did not cover lateral inferior head sites, making it difficult to identify activity from peri-insular sources. To test these data for potential ERP contributions from the insula, we first placed symmetric dipoles in the insular region of the BESA model we fit to the ERP. Next, we modeled the bilateral stimulus-related activities over occipito-parietal regions using bilaterally symmetric sources, fitting them in a preERN time segment in which their ERP activity peaked. Finally, two central dipoles were added to model the ERP data from the button press to the peak of the ERN. Different anatomical starting points for these dipoles were used to test the reliability of the solution. We modeled the remaining data until the residual variance across all 128 channels was below 5\% (see Section 3).

\section{Results}

\subsection{Behavioral analyses}

Analysis was conducted on the reaction time data with stimulus Compatibility (compatible, incompatible) and Response Type (correct, error) as within-subjects factors and subject Handedness as a between-subjects factor. Handedness was not a significant factor nor did it interact with any other factor. There was a significant Compatibility $\mathrm{X}$ Response Type interaction, $F(1,9)=35.7, P<0.001$. Simple effects analysis showed that for correct trials, incompatible responses were associated with longer RTs (366 $\pm 38 \mathrm{~ms})$ than compatible responses $(344 \pm 39 \mathrm{~ms})$, $F(1,10)=11.9, P<0.007$, and incompatible error responses, trials were associated with shorter RTs $(279 \pm 24 \mathrm{~ms})$ than correct incompatible trials $(330 \pm 33 \mathrm{~ms})$, $F(1,10)=53.4, P<0.001$. This later simple Response Type effect is explained by the fact that for incompatible trials, errors are essentially compatible responses, while for compatible trials, errors are incompatible responses.

An analysis was conducted for error count with Response Type as the within-subjects factor and Handedness as the between-subjects factor. Handedness did not interact with Compatibility. There was a main effect for compatibility; subjects made more errors when the response-stimulus mapping was incompatible, $62.6( \pm 45.8)(15.6 \%)$, than when the response-stimulus mapping was compatible, 26.1 $( \pm 6.6)(6.5 \%),(F(1,10)=14.8, P<0.004)$. These results are consistent with previous ERN studies using speeded response compatibility tasks.

Behavioral data were also compared in the Easy and Difficult cutoff conditions. As in a previous experiment (Luu et al., 2003), this feedback manipulation did not effect response speed or accuracy. Therefore, for the EEG analysis, data from both conditions were collapsed.

\subsection{Error-related ERP features}

Fig. $1 \mathrm{~A}-\mathrm{C}$ shows the grand average ERP data at channel Fcz. As can be seen in Fig. 1A, approximately $70 \mathrm{~ms}$ after the button press error responses are followed by a larger ERN negativity than correct responses, whose average also exhibits a small negativity as reported by a number of researchers (Luu et al., 2000b; Vidal et al., 2000). Fig. 1B shows the same ERP traces filtered with a $4-7 \mathrm{~Hz}$ (theta) bandpass. To more clearly show the effects of the thetabandpass filter, the theta-filtered and $0.1-30 \mathrm{~Hz}$ filtered error response ERP waveforms are superimposed in Fig. 1C. The effect of the filter is to isolate the ERP contents in the theta-frequency range. In addition to eliminating high frequency portions of the ERP, theta-band filtering also eliminates low frequency features. Both in the grand average and in many single-subject averages there is a slow negativity prior to the response (reminiscent of a motor preparatory potential or contingent negative variation) and a slow positivity after the response; for error trials this is termed Pe. The theta-bandpassed ERP accounts for $\sim 57 \%$ of the ERN peak amplitude and matches precisely the width of the wide-band ERN deflection at this channel. Fig. 1D-F show the topographic distributions of timevarying negativity at the 3 time points marked by the similarly labeled arrows in panel $1 \mathrm{~B}$, plus the location of the studied channel (Fcz).

To allow inspection of the ongoing EEG underlying the ERN, randomly selected (every 5th trial) wide-band singletrial EEG data at the same frontal midline scalp site (Fcz) from 4 subjects (selected at random) are presented in Figs. 2-5. Figs. 2A-5A show 10 such error trials, with (dark traces) and without (light traces) theta-bandpass filtering. Although the ongoing EEG waveforms are complex, the majority of the pre-response trial intervals contain appreciable theta activity that appears to visual inspection as irregular waves with a frequency varying in the $4-7 \mathrm{~Hz}$ range. Immediately after the button press, a negative-going EEG deflection can be seen clearly in most error trials in the unfiltered data. This ERN activity appears to arise in part from partial post-response phase consistency of theta activity, which appears to be more prevalent after the motor response. The phase alignment appears to be 
A

B

C
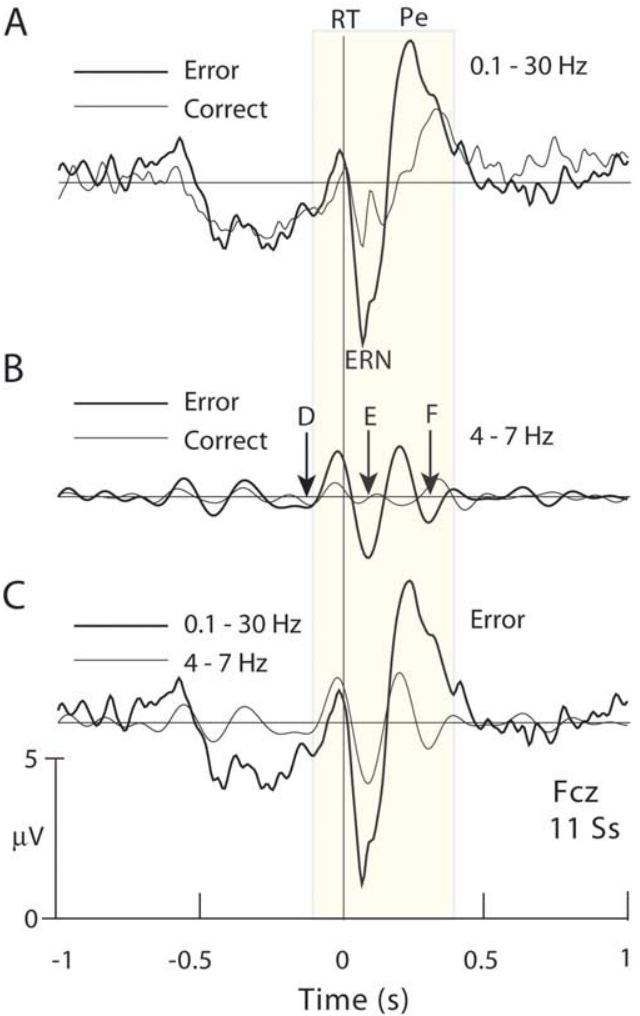

Theta-Band ERP

D

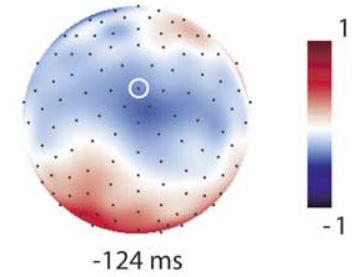

E

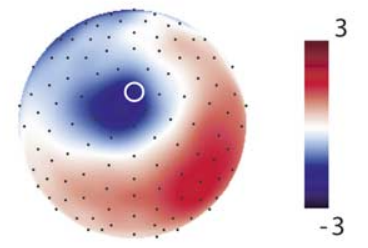

$72 \mathrm{~ms}$ $\mathrm{FCZ}$

$\mathrm{F}$

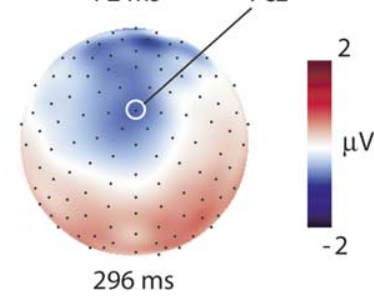

Fig. 1. (A) Grand average waveforms for correct and error responses from 11 subjects at site Fcz (circled in D). (B) Responses in A after theta-band (4-7 Hz) filtering. Arrows at the negativities around the ERN peak point to time points whose scalp maps are shown in D. (C) The grand-mean error-response ERP before and after theta-band filtering. (D) Interpolated scalp maps at negative peaks of the theta-band ERP in B. Orientation of the maps: from above looking down, nose pointing up. White circles: location of the Fcz electrode.

maximal at the dotted line after the button press that marks the time of the ERN peak.

Figs. 2B-5B show averages of the theta-band and wide-band filtered error trials for each subject. Note that in these individual subject averages about half the peak amplitude of the ERN again appears to be produced by theta activity, much of the remainder being associated with much higher-frequency activity. The succeeding Pe peak of the error-related ERP also is visible in some single trials (e.g. the 3rd and 9th trials in Fig. 2A, and most trials in Fig. 5A). The filtered data show that most of the energy of the $\mathrm{Pe}$ is not concentrated in the theta band but rather in the slower delta $(1-3 \mathrm{~Hz})$ band.

Effects that survive averaging across trials (whether theta filtered or not) represent activities that are, statistically, partially phase-locked to the time-locking experimental event (the button press). For example, a post-response peak, no matter how large that was alternatively positive and negative in successive trials would not be phase locked to the stimulus, and could not contribute reliably to the ERP. At other pre-response time points (for example, the point marked by the leftmost dashed lines in the colored panels of Figs. 2A-5A), theta activity in different trials has no preferred phase and therefore produces no ERP peak in the averaged data (cf. Figs. 2B-5B).

\subsection{Event-related EEG dynamics}

Fig. 6A shows the grand-average of the rectified bandpassed single-trial data at Fcz, representing total theta activity (i.e. either phase-locked or not phase-locked to the motor response). The increase in theta power in error trials lasts from approximately $100 \mathrm{~ms}$ before to $600 \mathrm{~ms}$ after the button press. Fig. 6B shows the mean time course of phaselocked theta amplitude in the grand-mean ERP. Peak theta amplitude occurs during the return phase of the ERN, at about $100 \mathrm{~ms}$. Fig. 6C shows the grand average time course of nonresponse-locked theta activity remaining after subtracting the single-subject response-locked ERP activity from each trial. It should be noted that Fig. 6B shows the peaked nature of the phase-locked activity (i.e. ERP). Fig. 6C shows the mean amplitude of single-trial theta activity remaining after removing the ERP from each trial. The data shown (in Fig. 6C) is scalloped at the theta frequency.

In both Fig. 6B and C, about $200 \mathrm{~ms}$ before the response the time courses of theta activity in correct and incorrect responses begin to diverge. At about the time of the button press, about $40 \%$ of the theta activity at Fcz becomes phaselocked to the response, creating the theta-band ERP and contributing to the ERN (Fig. 6B). The 'spiky' appearance of the error-response (dark) amplitude trace in Fig. 6B (see dots) 
A
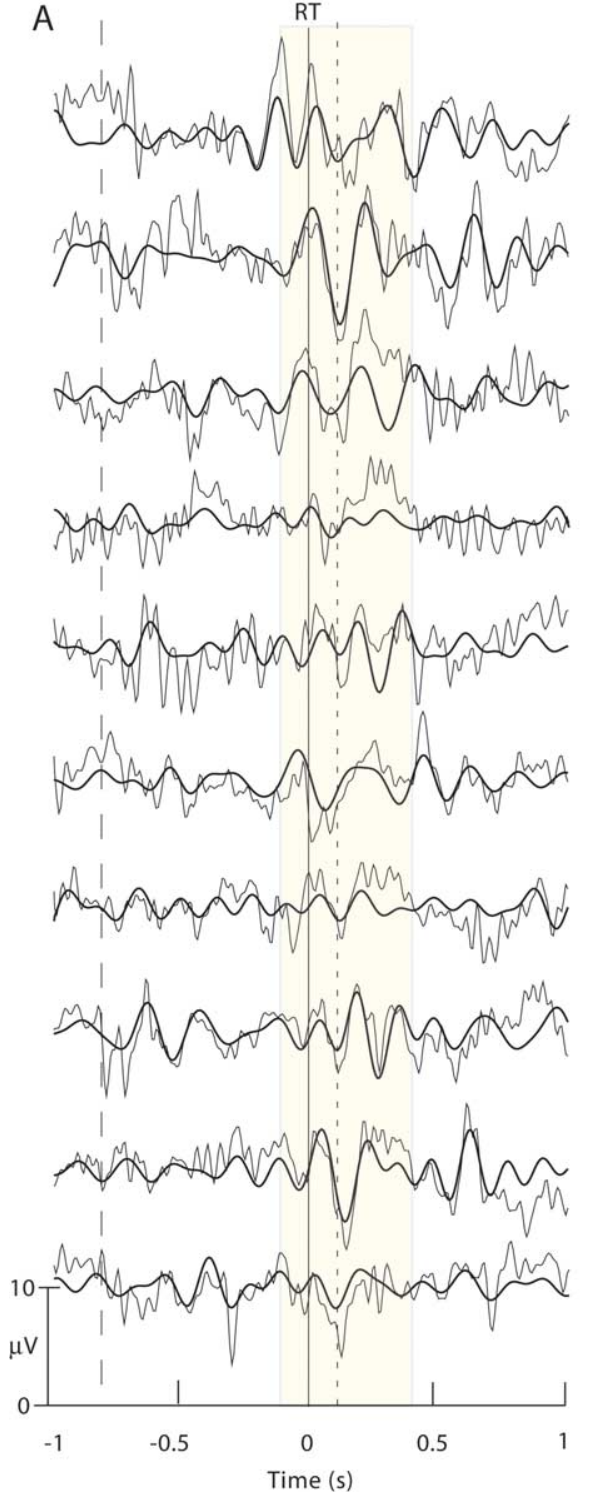

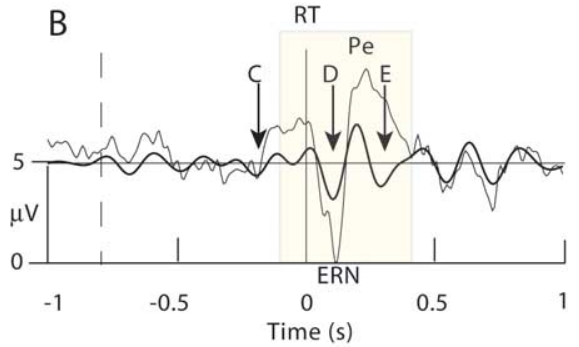

C

Theta-Band ERP

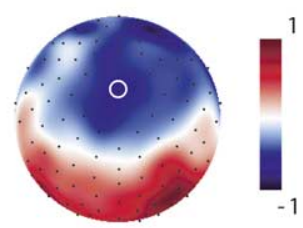

D

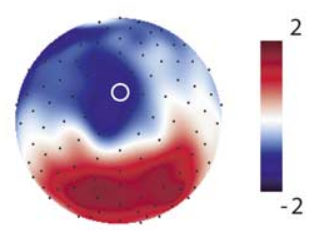

$\mathrm{E}$

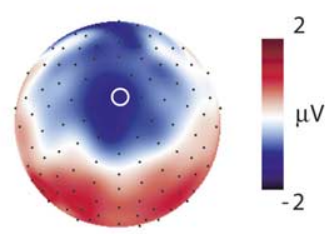

Fig. 2. Subject 1. (A) Waveforms of randomly selected single error response trials at site Fcz before (thin line) and after (thick line) theta-band filtering. Solid vertical line marks time of button press. Dashed line marks a arbitrarily selected time point before the response, dotted line marks the ERN peak latency. (B) Average of all the subject's error trials before (thin line) and after (thick line) theta-band filtering. Arrows at the negativities around the ERN are the time points shown in maps C-E. (C-E) Scalp maps of potential distribution at negative peaks of the theta-band ERP. White circles: site Fcz.

reflects the relative phase consistency, across subjects, of the theta-band ERPs. Subtracting the theta ERP from each trial thus produces marked 'dents' in the non-phase-locked amplitude time course (Fig. 6C, see dots). Both the response-locked theta that contributes to the ERN, and the theta-band EEG not phase-locked to the response are larger in error trials. However, the increase in non-phase-locked theta activity (Fig. 6C) following error responses occurs later and persists up to $400 \mathrm{~ms}$ after the response.

\subsection{Statistical analysis}

Paired one-tailed $t$ tests were conducted on the thetabandpassed data to confirm that error responses at the Fcz sensor were associated with a larger ensuing negative potential (the ERN) than correct responses. The ERN was measured as the average amplitude of the voltage data in the interval between 50 and $100 \mathrm{~ms}$ after button press relative to the average potential of the -400 to -200 ms interval prior to the button press. As expected, error responses were associated with a larger negativity than correct responses $(t(10)=5.18$, $P<0.001)$, replicating previous ERN findings.

To statistically analyze the non-phase-locked data, we took the average of non-phase-locked theta amplitude (Fig. 6C) in successive $100 \mathrm{~ms}$ intervals from $200 \mathrm{~ms}$ before button press to $400 \mathrm{~ms}$ after button press. For this analysis, fMT was measured at two frontal midline sites ( $\mathrm{Fz}$ and $\mathrm{Fcz}$ ), again referenced to its average value in the interval $400-200 \mathrm{~ms}$ before the button press. A repeatedmeasures ANOVA with 3 factors, Time Interval, Site 


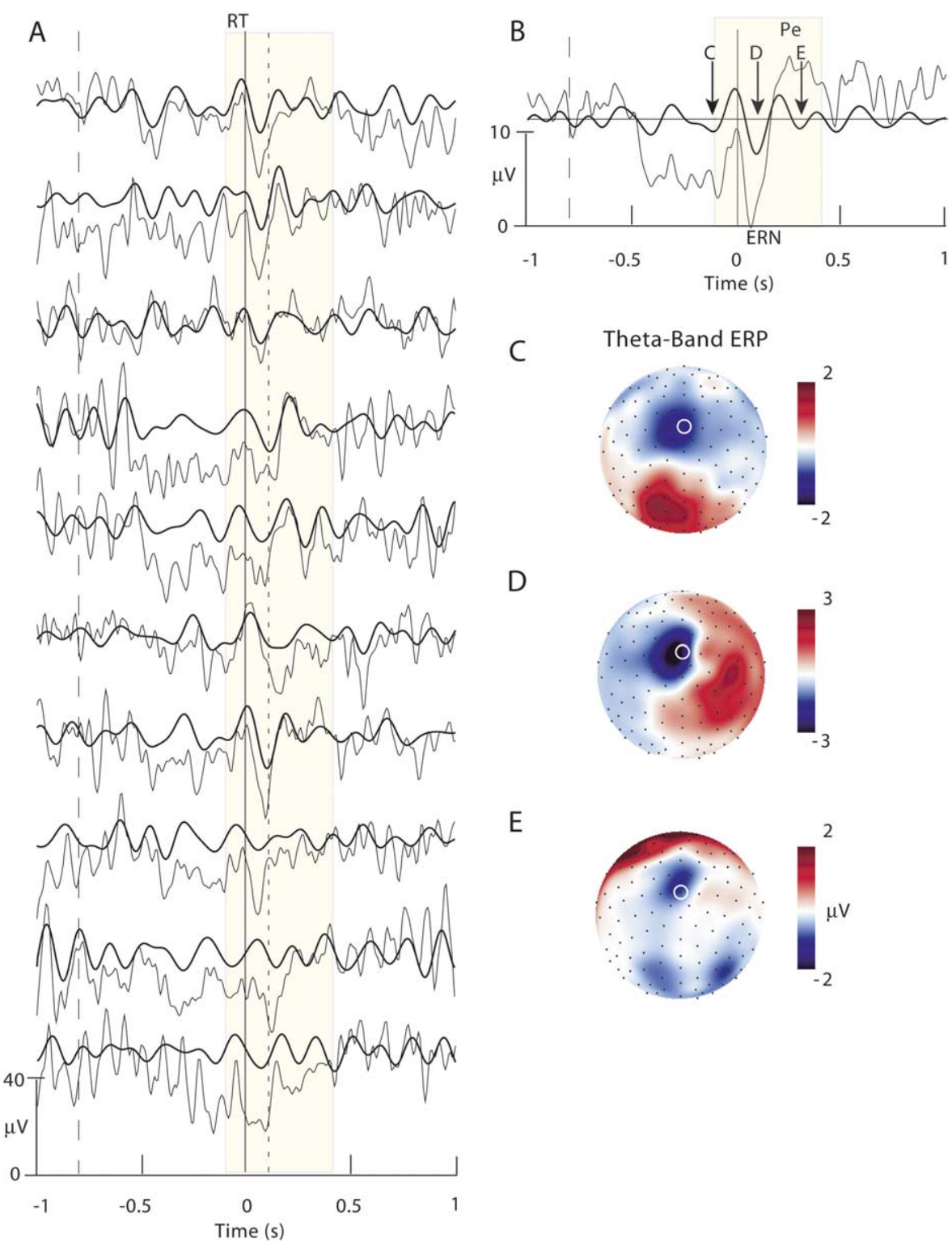

Fig. 3. Randomly selected single trials for a second subject, as in Fig. 2.

(Fz, Fcz) and Response Type (Correct, Error), was conducted to examine (1) whether error responses were associated with significant changes in non-phase-locked fMT compared to correct responses, and (2) whether this fMT difference extended beyond the ERN time window. The latter test is relevant for determining whether the phaselocked ERN waveform could be said to contribute much or all of the extra energy at the theta frequency independent of any non-phase-locked theta-band process. Geisser-Greenhouse correction was applied where appropriate.

In all intervals, the Site factor exhibited no statistically significant differences. However, the analysis revealed a significant Time Interval by Response Type interaction, $F(5,50)=10.8, \quad P<0.001 \quad$ (Giesser-Greenhouse epsilon $=0.468$, see Fig. 7). To explore the interaction, Response Type was analyzed for each time interval.
The response-type difference in theta amplitude was not significant in the $[-200 \mathrm{~ms},-100 \mathrm{~ms}]$ interval. Between $-100 \mathrm{~ms}$ and the button press, there was a trend for correct responses to be associated with more non-phase-locked theta activity $(F(1,10)=4.0, P<0.08)$. In the interval between button press and $200 \mathrm{~ms}$ post-button press, the analysis revealed no statistically significant difference in non-phase-locked theta amplitude. As Fig. 7 shows, after the button press, in both the [200 ms, $300 \mathrm{~ms}$ ] and [300 ms, $400 \mathrm{~ms}$ ] post-response intervals, non-phase-locked theta amplitude was larger following error responses $(F(1,10)=8.3, P<0.02$ and $F(1,10)=35.3, P<0.001$, respectively).

Fig. 8a shows the scalp distribution of grand-mean amplitude of non-phase-locked theta activity before, at and after the ERN peak. The EEG theta amplitude maximum at 


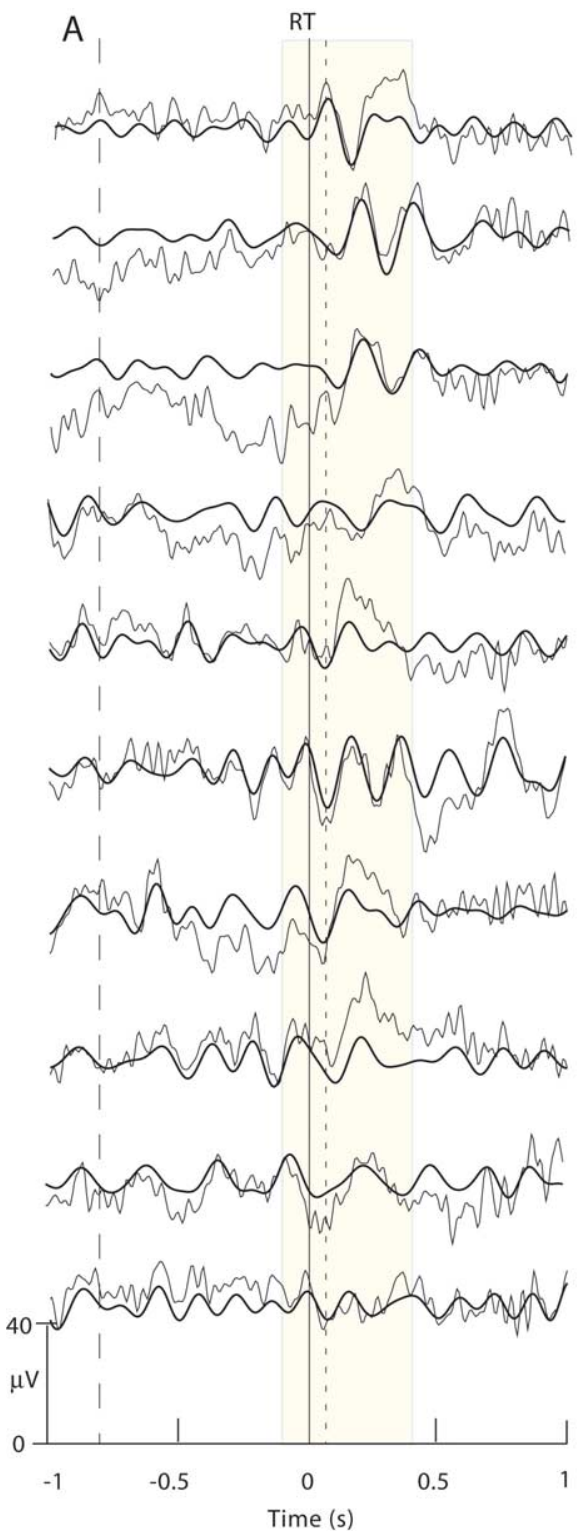

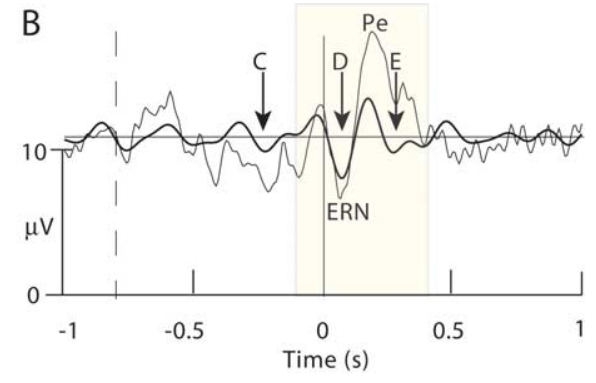

C

Theta-Band ERP

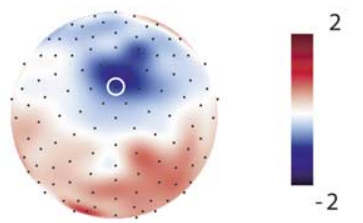

D

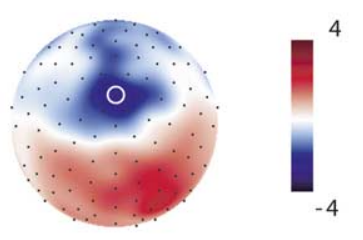

$E$

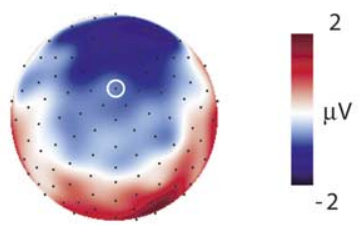

Fig. 4. Randomly selected single trials for a 3rd subject, as in Fig. 2.

about $100 \mathrm{~ms}$ is at mediofrontal sites, consistent with previous research reporting the distribution of fMT (Gevins et al., 1997). During the later period of theta excess in the error trials (250-400 ms, cf. Fig. 6C), non-phaselocked theta activity remains largest along the mediofrontal electrode sites. At the ERN peak, the spatial theta-EEG peak (Fig. 8) is anterior to the center of the ERN scalp distribution (Fig. 1B). It should also be noted that distribution of the midline theta activity does not topographically overlap with the distribution of the Pe (Fig. 8b).

\subsection{ERP source analysis}

Is the ERN largely a result of partial phase-locking of theta-band EEG activity? It might be proposed that the apparently oscillatory features of the filtered eventrelated scalp data (e.g. Figs. 2-5) are artifacts of successive, transient monophasic activations of multiple cortical sources whose volume conducted activities are superposed at the scalp electrodes at near-equal intervals so as to form apparently oscillatory sequences. In this view, each peak might have a different scalp and source distributions, and each underlying cortical source would contribute to just one ERP peak. To evaluate this possibility, we performed equivalent dipole source analysis of the error-response ERP waveform. In equivalent dipole models, each dipole is essentially a spatial filter modeling the scalp projection of neural activity from a cortical (or other) source region to the scalp electrodes (Scherg and Berg, 1996). The multiple dipole model used in the present source analysis consisted 


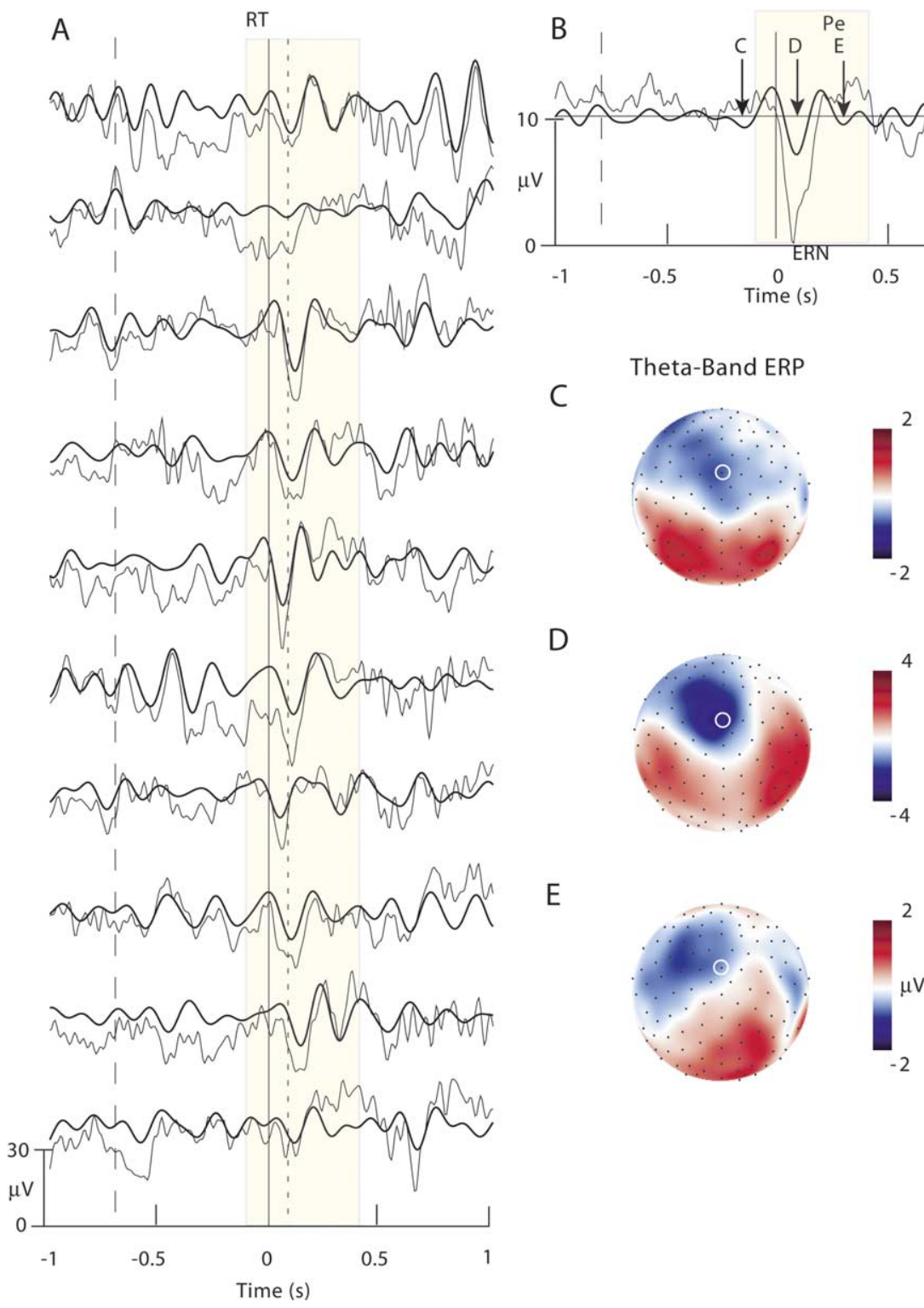

Fig. 5. Randomly selected single trials for a 4th subject, as in Fig. 2.

of 7 equivalent-dipole signal sources: 3 midline plus two bilateral pairs, insular, and temporal-parietal. After training by iterative gradient descent, starting from their hypothesized approximate locations, the 7-dipole model accounted for approximately $95 \%$ of the variance of the 128-channel grand-average response through the time interval from $200 \mathrm{~ms}$ before to $500 \mathrm{~ms}$ after the error response.

The 3 modeled midline dipole locations and orientations are shown in Fig. 9A, superimposed on an average MRI brain image. Unlike a previous report (Luu et al., 2003) in which only two midline dipoles were required to model the ERN, the present data required 3 midline dipoles for a stable solution. Dipole 1 was located in the vicinity of the marginal branch of the cingulate sulcus, Dipole 2 in the region of the midcingulate shown to be engaged in error monitoring and feedback evaluation in several experiments (see Tucker et al., 2004). Dipole 3 was located in the rostral ACC at the level of the genu of the corpus callosum, consistent with Brodmann area 32. Fig. 9C shows the projections of each of the equivalent dipoles to the electrode array (common units, relative $\mu \mathrm{V})$, together with the total theta-band ERP at the Fcz peak of the ERN, which they model. Fig. 9B shows the theta-band limited activity time courses (or moments) of these dipoles. All 3 midline dipoles have larger phaselocked theta-ERP activity following errors, particularly near the ERN peak (occurring, for site Fcz, at the dashed line). Note that, here, none of the 3 modeled midline dipoles occupies the dorsal anterior cingulate region per se, and might thus not account for the more anterior scalp distribution of the 'excess' non-phase-locked theta after the button press (Fig. 8). 

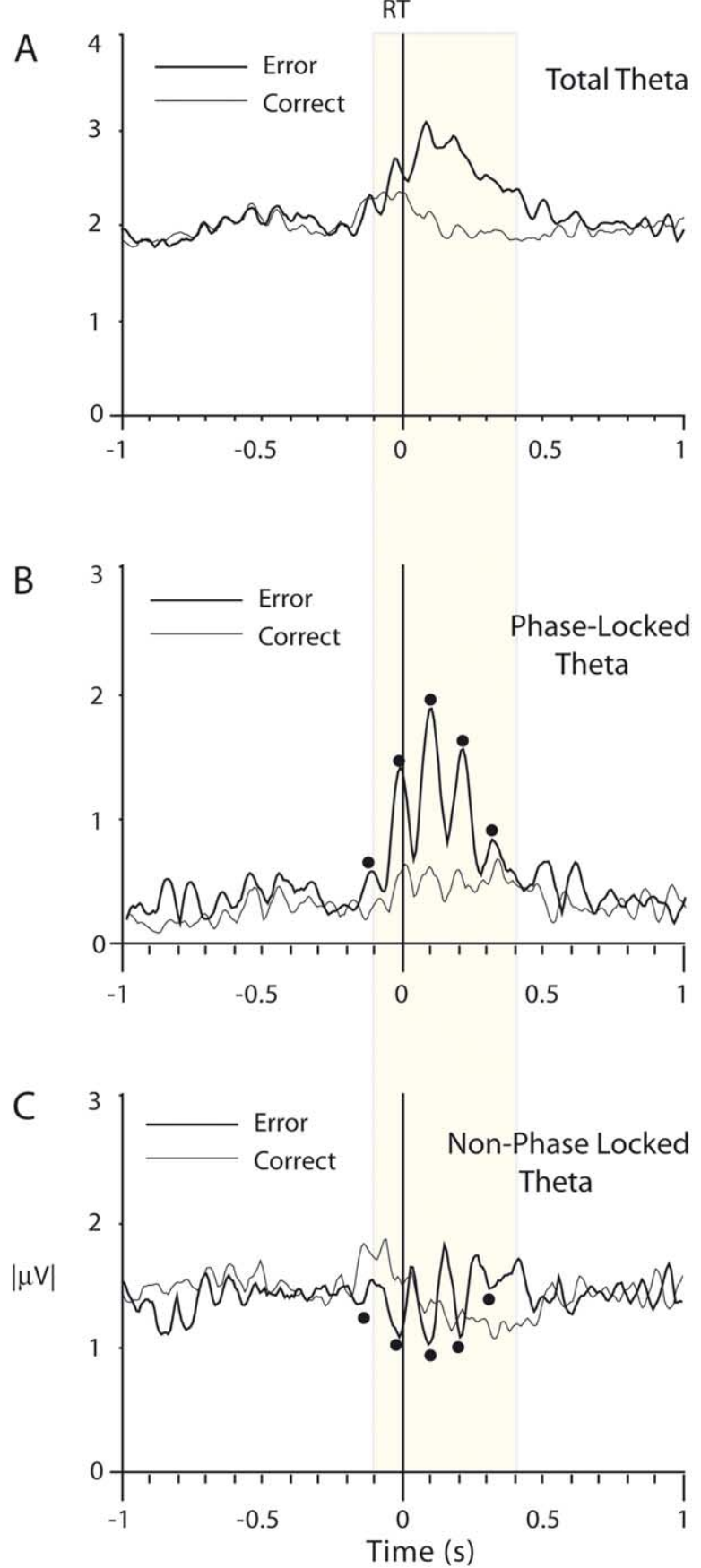

Fig. 6. (A) Grand average time courses of mean rectified, theta-band amplitude at Fcz in correct and error response trials. (B) Grand average time courses of mean rectified, theta-band amplitude in the single-subject averaged correct and error response ERPs at Fcz. These data measure only the theta activity at Fcz that was phase-locked to the motor response. (C) Grand average time courses of rectified, theta-band amplitude at Fcz in correct and error response trials after subtracting the relevant subject-mean ERP from each trial. These data represent theta activity at Fcz not phase locked to the button press.

Locations, scalp projections and activity time courses of the two modeled insular sources are consistent with the previously reported 256-channel results. The peaks of the activity of the two insular sources do not occur concurrently with the peak of the ERN. Because of this fact, and their lateral orientations, the insular model sources do not contribute substantially to the fronto-central topography of the scalp ERN.

\section{Discussion}

\subsection{ERN and theta-band EEG}

The results presented here are consistent with the hypothesis that the ERN in averaged data emerges in part from the stronger phase-locking of midline EEG activities to the motor response following error responses, particularly in the theta-frequency band. Luu and Tucker (2001) observed energy in the theta-filtered ERP over the frontal midline and over motor cortex for both correct and incorrect trials. The midline theta ERP, containing the majority of the ERN, was correlated with the average waveform recorded over motor cortex when errors were committed. Makeig et al. (2002) noted that for each subject tested, two or more independent components of the unaveraged EEG data from an ERN experiment showed increased theta amplitude following the motor response which was larger and appeared more phase consistent following error responses.

The present study extends the previous ERP analysis in several ways. Here, the EEG data were filtered with a $4-7 \mathrm{~Hz}$ bandpass prior to epoch segmentation and averaging. This allowed identification of ERN-related theta-band EEG activity in randomly selected collections of single trials. By using a zero-phase distortion FIR filter, the phase of the data, and so the latencies of the data peaks, were preserved, and the relation of oscillatory EEG dynamics to the partially phasealigned signal producing the theta-band limited averaged ERP was visible in single-trial subsets. By filtering the EEG data before averaging, the single-trial traces could be examined for theta wave patterns not phase-locked to the motor response, which thus did not survive averaging. Inspection of single-trial traces did not reveal large distortions introduced by filter ringing at the edges of the ERN. Finally, by separating the phase-locked and non-phaselocked features of the theta-band activity, the relation between the dynamics of 'background' theta-EEG activity and the ERN feature of the average ERP could be explored.

As shown in Figs. 2-7, the negative peak of the ERN in the response-locked averaged waveforms was not the only feature discriminating error from correct response trials. Rather, following error responses, in some trials there was a transient increase, relative to the pre-response baseline, in the amplitude of theta activity. Careful inspection of filtered and unfiltered single trials (Figs. 2-5) shows intermittent theta activity both before and after the response in both correct and error trials. The theta-band portion of the ERN in the trial average appeared to emerge from a brief and partially consistent phase-alignment of the increased theta activity following the motor response. While the partial 


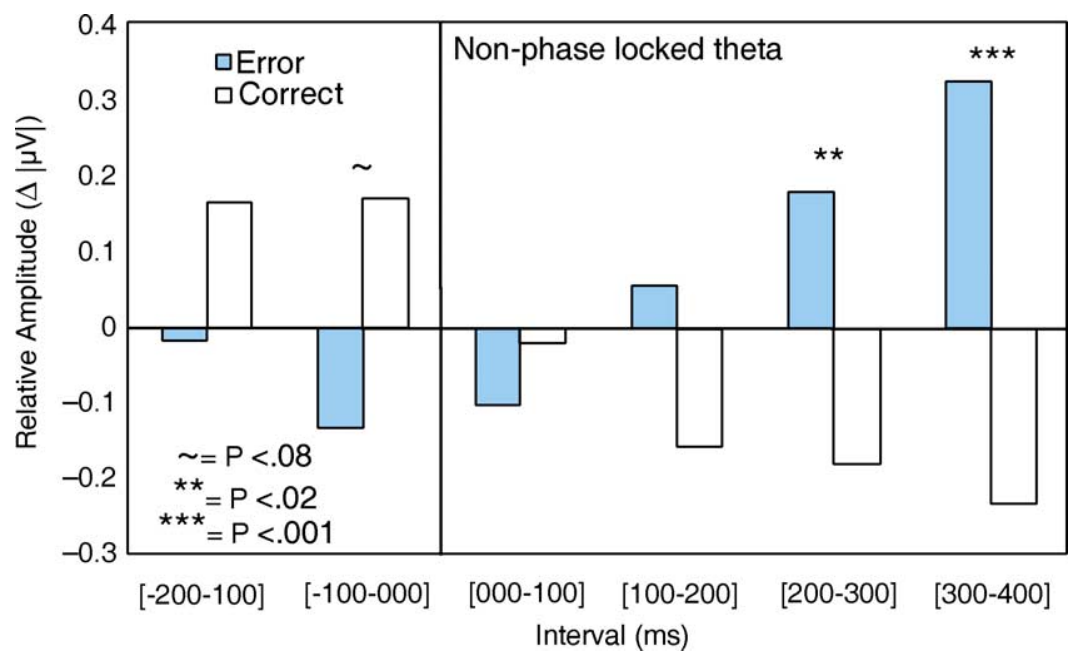

Fig. 7. Mean non-phase-locked theta amplitudes in correct and error response trials (shown in Fig. 6C) at $100 \mathrm{~ms}$ intervals starting $200 \mathrm{~ms}$ before the button press, with significant differences indicated.

phase-locking and extended theta amplitude increase both contribute to a negative-going deflection that survives averaging to form the negative wave recognized as the ERN, the partial phase-locking is primarily responsible. Note that the amplitude of the theta-band ERP (Fig. 6B) is smaller than baseline theta-EEG amplitude (Fig. 6A).

To date, ERN research has studied averaged (usually, grand-averaged) ERP data. However, a recent report by Brázdil et al. (2002) presented data averaged over very few trials. Inspection of their Fig. 4 shows that the ERN appears to be closely associated with brief bursts of theta activity, similar to the association seen in the unaveraged trials of Figs. 2-5. The theta-band error-response locked ERP (Fig. 1B) also strongly resembles a two-cycle theta-burst feature that dominates the wide-band single-trial data and responselocked ERP at frontocentral sites in a visual selective attention task (Makeig et al., 2004; Makeig et al., submitted). In this report, the authors applied ICA decomposition to the unaveraged EEG data and found at least 4 classes of EEG processes that contributed to the theta-burst ERP-two frontal midline clusters plus left and right mu-rhythm processes localizing to or near hand somatomotor cortex.

In the present data, both the theta-band ERN and the amplitude of non-phase-locked post-response EEG were maximal at medial frontal recording sites, consistent with the notion that the ERN emerges in part from phasealignment of fMT EEG processes. The scalp distributions of the theta-band ERN and remaining theta-band EEG were somewhat different, suggesting that different theta-producing EEG sources may be phase-locked to the motor response of different extents, thus contributing in different proportions to the phase-locked (ERP) and non-phaselocked data portions.

Although the ERN appears after the motor response, Figs. 1B and 6B suggest that phase consistency of EEG theta activity may begin before the button press.
A

$$
\begin{aligned}
& \text { Non-Phase Locked Theta } \\
& \text { (4- } 7 \mathrm{~Hz})
\end{aligned}
$$
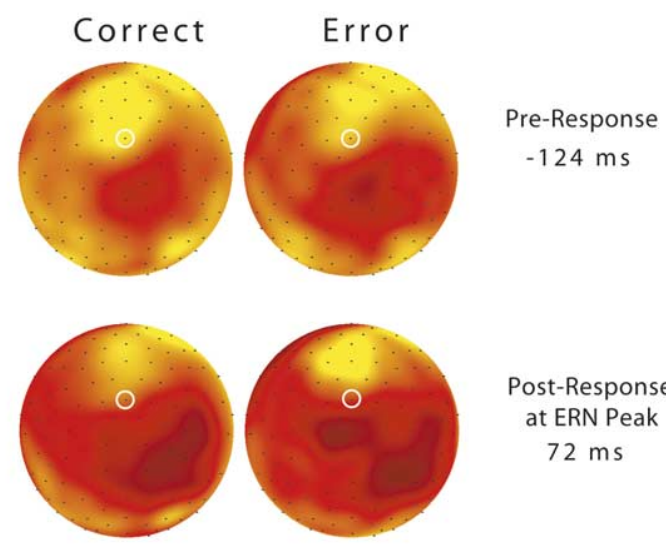

Post-Response at ERN Peak $72 \mathrm{~ms}$
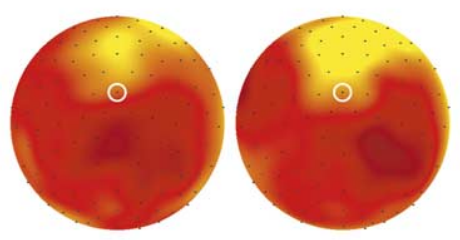

Post-Response at Pe Peak $222 \mathrm{~ms}$
B

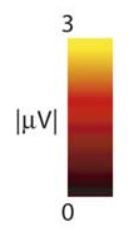

Phase-Locked Wide-Band $(0.1-30 \mathrm{~Hz})$

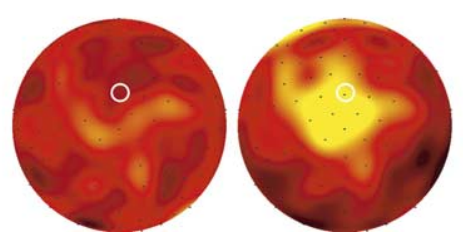

ERP Amplitude at Pe Peak $222 \mathrm{~ms}$
Fig. 8. (A) Scalp maps showing the distribution of mean non-phase-locked theta-band amplitude in correct and error trials at 3 time points. (B) Grandmean ERP scalp map at the Pe peak from the unfiltered error response (Fig. 1A). White circles: location of site Fcz. 
A

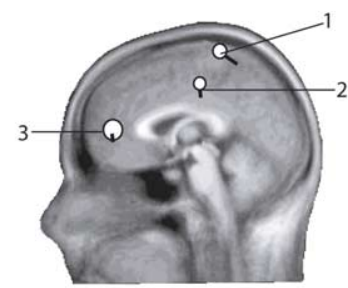

B

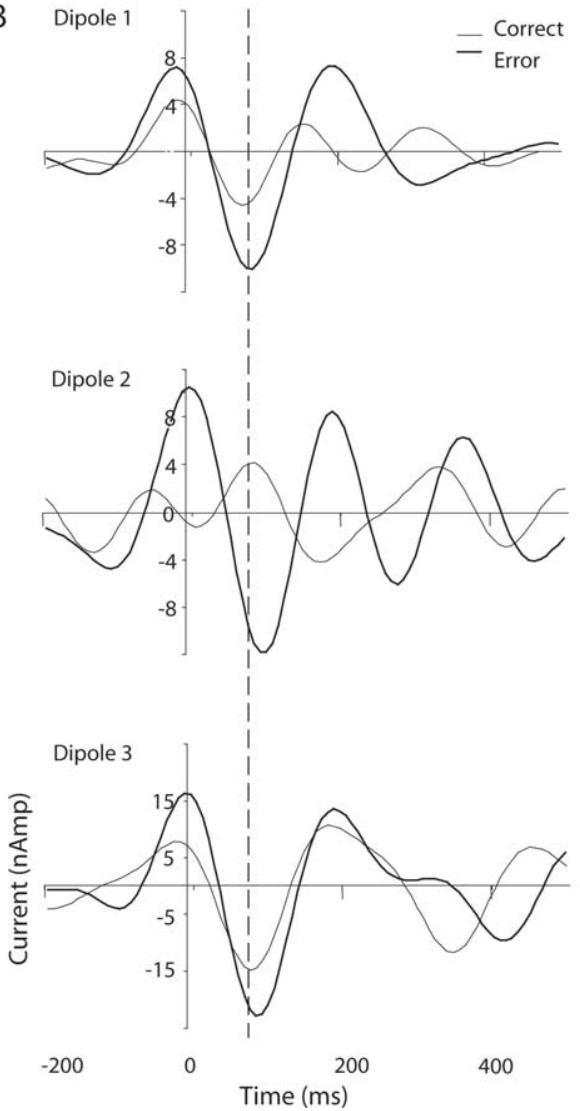

C
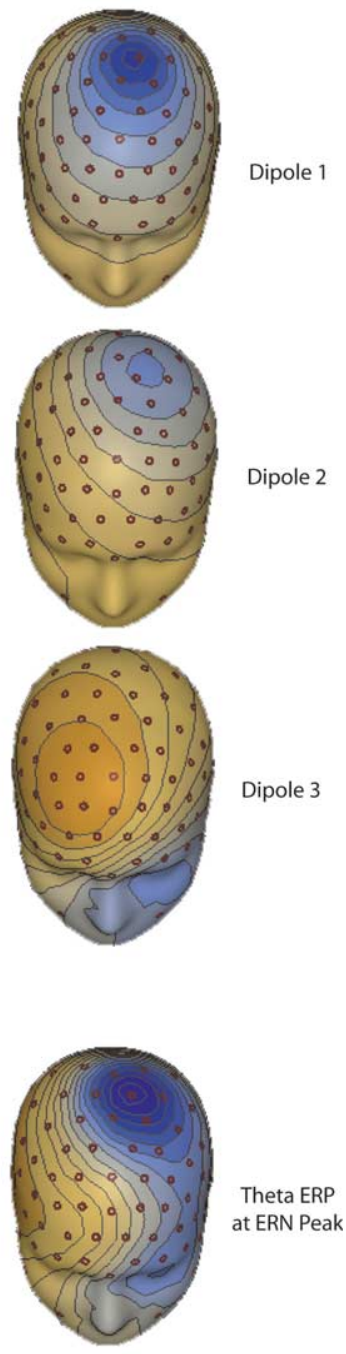

Fig. 9. (A) Modeled locations of 3 midline dipoles fit to the theta-band correct and error response-locked grand mean ERPs and registered to an average MR image. (B) Source ERP waveforms for the 3 midline dipoles. (C) Scalp maps showing the relative projections of the 3 dipoles, above a scalp map of the grand mean theta-band error response locked ERP at the ERN peak.

In the phase-locked data, $100 \mathrm{~ms}$ before error responses fMT bursts became partially phase-aligned to the button press more strongly than before correct responses (Figs. 1B and 6B). This is consistent with the interpretation that the theta-band portion of the ERN sums activity of EEG processes contributing to the fMT increase, rather than being a discrete, monophasic evoked response whose generators are unrelated to the generation of other EEG activity.

During the $200 \mathrm{~ms}$ interval following error responses, mean non-phase-locked theta amplitude at the analysis site (Fcz) was not significantly larger than following correct responses (Fig. 6C). However, the time course of nonphase-locked theta amplitude in this interval naturally occurred near zero crossings in the theta ERP. If the ERN were simply a transient added to unperturbed, ongoing EEG following error responses, then the time course of total theta amplitude (Fig. 6A) might be expected to be scalloped, and removing the ERN from each EEG trial might be expected to leave a relatively smooth non-phase-locked theta amplitude curve like the smooth total amplitude trajectory for error trials in Fig. 6A. Instead, the jagged characters of the phase-locked and non-phase-locked post-error response amplitude trajectories (Fig. 6B and C) and the smooth character of the total theta increase (Fig. 6A) together suggest that our separation of the data here into phaselocked and non-phase-locked components does not produce two physiologically distinct data subsets.

One might object that our ERP subtraction method did not allow for between-trial amplitude and phase or latency differences. A much more flexible or 'generalized' method for separating ERP from 'EEG' data in single trials might regress out the optimally time-shifted subject-mean ERN from each trial, allowing for both amplitude and latency 
differences between trials. However, as the theta-band ERP (Fig. 1B) resembles a generic two-cycle theta wavelet, such a generalized regression method might essentially model and remove all theta band EEG from the single-trial datain effect redefining the EEG as a phase and amplitude 'generalized ERP' This would vitiate the distinction between phase-locked and non-phase-locked data, and thus between the ERP and the ongoing EEG, and would essentially accede to our claim, here, that the average ERN and post-response theta-band EEG bursts in single trials are intimately related.

Might some other single-trial ERP separation method be more reasonable? Unfortunately, the ERP (and thus, the ERN) is defined as a trial average, and is in general not defined in single trials (Makeig et al., 2004). This truism is particularly important for ERP features with oscillatory content. If:

1. The ERN and fMT reach the same scalp channels through volume conduction,

2. The ERN and fMT both have much of their energy in the theta band, and

3. The ERN occurs on the up-slope of an $800 \mathrm{~ms}$ period of increased fMT activity that is not wholly accounted for by the ERP, and if

4. The ERN is not defined in single trials,

then it is unreasonable to reject the possibility, at least, that the theta-band energy in the theta-band content of the ERN is intimately linked to perturbations of fMT amplitude and phase in single trials following error responses.

That non-phase-locked theta activity differs following correct and error responses challenges a common assumption that non-phase-locked EEG, in this case fMT activity, eliminated by the averaging process is merely background noise. Clearly, the considerable research literature linking fMT to concentration does not support such a dismissal (Givens, 1996; Mizuki et al., 1980; Sasaki et al., 1996; Slobounov et al., 2000). However, further research is required to definitively address this issue. Proving that no such link exists between ERN and fMT may be difficult, particularly without considering relationships between the spatiotemporal distributions of the ERN and fMT. Single scalp-channel data are the sum of potentials volume conducted from many cortical domains. Most desirable, therefore, would be separation of ongoing EEG activity into contributions from separate cortical source regions. This would allow noninvasive study of event-related brain dynamics within the brain, without requiring the possibly artificial separation (as here) of phase-locked and non-phase-locked data portions.

\subsection{ERN and fMT sources}

The EEG is a spatiotemporal sum of volume-conducted potentials originating from multiple cortical sourcesdomains or patches whose electrical activity is partially synchronized and suitably oriented to reach the scalp electrodes through volume conduction. The biophysical inverse problem of determining the physical source distribution of a given set of scalp maps is underdetermined. Therefore, additional information or hypotheses are required to fit a source model to EEG data. The present equivalent dipole source model was derived from a mixture of exploratory and confirmatory approaches. Previous research using a purely exploratory approach has also reported ACC territory sources for the ERN (Dehaene et al., 1994; Luu et al., 2000a; van Veen and Carter, 2002).

Because of the spatiotemporal complexity of the grandaverage ERP data, multiple midline sources are necessary to model the grand-average ERN data (Luu et al., 2003). Rüsseler et al. (2003) also found that a two-dipole solution, one in the ACC and another in the posterior cingulate cortex, was required to account for the activity of the ERN. Consistent with these prior findings, equivalent dipole source analysis of the present data required multiple cortical sources to model the theta-band ERN, and the waveforms associated with the theta-band ERP contributions of these dipoles showed a two (or more) cycle pattern of phase locking (most likely partial) to the button press (Fig. 9).

The observation that the portions of the band-limited ERP accounted for by the modeled dipole sources also exhibited oscillatory features in single trials (Figs. 2-5) provides further though limited support for the hypothesis that theta-band activity plays a role in self-monitoring processes occurring in the cingulate gyrus and/or adjacent medial cortex. Although transient, monophasic deflections in sensory cortical areas, conventionally considered to generate ERPs, might occur in sensorimotor paradigms, the ERN appears to be related to transient oscillatory fMT EEG processes. It seems likely that similar responses, for example, the medial frontal negativity or MFN following evaluative feedback (Gehring and Willoughby, 2002; Luu et al., 2003), may have similar origins.

However, hypothesis-guided multi-dipole models of ERP dynamics cannot claim to be unique. In particular, fitting only the relatively small portion of the theta-EEG energy captured by the ERP, as we have done here, may strongly bias the source model. While our model demonstrates that there must be multiple near-midline sources of the theta-band portion of the ERN, the full distribution of those sources may not be captured by a model trained only on the ERP. In particular, the 3 midline dipoles in our model (Fig. 9A) appear to be rostral and posterior, respectively, to the dorsal anterior cingulate cortex territory often shown to be active in response error-related fMRI comparisons (Garavan et al., 2004; Ullsperger and von Cramon, 2001), and to the $(\mathrm{Fz})$ maximum of the non-phase-locked theta amplitude increase (Fig. 8).

Although not a source analysis method, the Laplacian, a second derivative measure, estimates scalp current density (CSD). The Lapalacian is most sensitive to superficial, radially oriented sources. Visual inspection of CSD can be 
useful for checking the plausibility of computed source locations. At the suggestion of a reviewer, we examined the scalp distribution of the CSD for each dipole. In order of CSD strength, Dipole 1 showed a clear current source sink along mediofrontal sites followed by the activity of Dipole 2 at frontal recordings sites. In contrast, activity of deeper Dipole 2 contributed very minimally to the CSD, although it did contribute to the overall scalp voltage potential distribution of the ERN. When we moved Dipole 2 forward to more anterior ACC, the CSD of this source remained weak, consistent with previous research using the Laplacian to measure the ERN (Vidal et al., 2000). Interestingly, Vidal et al. (2003) have recently reported that in the Laplacian derivation the ERN can be observed as theta oscillations along mediofrontal recording sites, consistent with our previous resports (Luu and Tucker, 2001; Makeig et al., 2002) and the present study.

Sources of theta activity in EEG and ERPs need not be limited to the frontal midline. Because the 128-channel array used here did not adequately sample potentials from the inferior surface of the head, we drew upon a previous 256-channel EEG model (Tucker et al., 2004) and fMRI studies (Menon et al., 2001) to seed dipoles in or near the bilateral insular regions to act as spatial filters for activity originating there. The orientations of these dipoles were then fit to the data and accounted for residual energy in the scalp potential that was not well-described by the other model sources. While the contribution of the peri-insular sources to the ERN itself was minimal, the modeled dipole theta-band ERP contributions differed between correct and error responses, as in the Tucker et al. study. Further studies will be required to illumine the contribution of the insula to error evaluation and action regulation.

\section{Conclusion}

If it proves possible to clarify the contribution of thetaEEG activity to the ERN, there will be a number of interesting theoretical implications for the functional interpretation of the ERN. With respect to the error monitoring view of the ERN, in primates the most reliable method for eliciting hippocampal theta is withholding an expected reward (Crowne and Radcliffe, 1975). Furthermore, hippocampal theta is more prevalent in the early stages of learning, whereas it habituates in the later stages (Pickenhain and Klingberg, 1967). These properties are very similar to error activity recorded in single cells (Niki and Watanabe, 1979) and to error potentials recorded in primate ACC (Gemba et al., 1986). These facts, as well as the fact that theta-EEG activity indexes different stages of learning (Powell and Joseph, 1974), may foster a deeper understanding of the role of the ACC in error monitoring and action control.

It is difficult to judge the implications of the present findings for response conflict monitoring and other interpretations of the ERN. Very likely, both response conflict monitoring and more general error monitoring are closely linked to affective control processes. Critchely et al. (2003) found overlapping ACC fMRI activations following response conflicts and simple motor acts, and reported that the common activation was correlated with sympathetic autonomic nervous system responses. Also, Kubota and colleagues (2001) demonstrated that fMT increases are correlated with heart rate changes. Therefore, the autonomic visceral system activation associated with ACC activity may, in part, help explain why conflicting response demands and error responses are associated with larger fMRI BOLD signals and ERN amplitude (Luu and Posner, 2003). The recent demonstration of coupled theta activity in hippocampus and amygdala following a conditioned fear cue in animals (Seidenbecher et al., 2003) suggests the possibility of a more general role for cortical theta dynamics in affective cognition.

\section{Acknowledgements}

This research was supported by National Institutes of Mental Health grant Nos MH42129 and MH42669 and DARPA grant No. 03SB1-0127 to D.M.T. and P.L. and the Swartz Foundation (S. M.).

\section{References}

Asada H, Fukuda Y, Tsunoda S, Yamaguchi M, Tonoike M. Frontal midline theta rhythms reflect alternative activation of prefrontal cortex and anterior cingulate cortex in humans. Neurosci Lett 1999;274: 29-32.

Basar E. Brain function and oscillations. Berlin: Springer; 1998.

Basar-Eroglu C, Basar E, Demiralp T, Schürmann M. P300-response: possible psychophysiological correlations in delta and theta frequency channels: a review. Int J Psychophysiol 1992;13:161-79.

Brázdil M, Roman R, Falkenstein M, Daniel P, Jurák P, Rektor I. Error processing: evidence from intracerebral ERP recordings. Exp Brain Res 2002;146:460-6.

Carter CS, Braver TS, Barch DM, Botvinick MM, Noll D, Cohen JD. Anterior cingulate cortex, error detection, and the online monitoring of performance. Science 1998;280:747-9.

Critchley HD, Mathias CJ, Josephs O, O’Doherty J, Zanini S, Dewar B-K, Cipolotti L, Shallice T, Dolan RJ. Human cingulate cortex and autonomic control: converging neuroimaging and clinical evidence. Brain 2003;126:2139-52.

Crowne DP, Radcliffe DD. Some characteristics and functional relations of the electrical activity of the primate hippocampus and a hypothesis of hippocampal function. In: Isaacson RL, Pribram KH, editors. The hippocampus: neurophysiology and behavior, vol. 2. New York: Plenum Press; 1975. p. 185-206.

Dehaene S, Posner MI, Tucker DM. Localization of a neural system for error detection and compensation. Psychol Sci 1994;5:303-5.

Derryberry D. Right hemisphere sensitivity to feedback. Neuropsychologia 1990;28:1261-71.

Derryberry D. The immediate effects of positive and negative feedback signals. J Pers Soc Psychol 1991;61:267-78.

Falkenstein M, Hohnsbein J, Hoormann J, Blanke L. Effects of crossmodal divided attention on late ERP components. II. Error processing in 
choice reaction tasks. Electroencephalogr Clin Neurophysiol 1991;78: 447-55

Garavan H, Ross TJ, Kaufman J, Stein EA. A midline rostral-caudal axis for error processing and response conflict monitoring. Neuroimage 2003;20:1132-9.

Gehring WJ, Willoughby AR. The medial frontal cortex and the rapid processing of monetary gains and losses. Science 2002;295: 2279-82.

Gehring WJ, Goss B, Coles MGH, Meyer DE, Donchin E. A neural system for error detection and compensation. Psychol Sci 1993;4:385-90.

Gemba H, Sasaki K, Brooks VB. 'Error' potentials in limbic cortex (anterior cingulate area 24) of monkeys during motor learning. Neurosci Lett 1986;70:223-7.

Gevins AS, Cutillo BA, Bressler SL, Morgan NH, White RM, Illes J, Greer D. Event-related covariances during a bimanual visuomotor task. II. Preparation and feedback. Electroencephalogr Clin Neurophysiol 1989; 74:147-60.

Gevins A, Smith ME, McEvoy L, Yu D. High-resolution EEG mapping of cortical activation related to working memory: effects of task difficulty, type of processing, and practice. Cereb Cortex 1997;7:374-85.

Givens B. Stimulus-evoked resetting of the dentate theta rhythm: relation to working memory. Neuroreport 1996;8:159-63.

Gratton G, Coles MG, Donchin E. A new method for off-line removal of ocular artifact. Electroencephalogr Clin Neurophysiol 1983;55: 468-84.

Kubota Y, Sato W, Toichi M, Murai T, Okada T, Hayashi A, Sengoku A. Frontal midline theta is correlated with cardiac autonomic activities during the performance of an attention demanding meditation procedure. Cogn Brain Res 2001;11:281-7.

Luu P, Posner MI. Anterior cingulate regulation of sympathetic activity. Brain 2003;126:2119-20.

Luu P, Tucker DM. Regulating action: alternating activation of human prefrontal and motor cortical networks. Clin Neurophysiol 2001;112: 1295-306.

Luu P, Tucker DM. Self-regulation and the executive functions: electrophysiological clues. In: Zani A, Preverbio AM, editors. The cognitive electrophysiology of mind and brain, San Diego: Academic Press; 2003. p. 199-223.

Luu P, Collins P, Tucker DM. Mood, personality, and self-monitoring: negative affect and emotionality in relation to frontal lobe mechanisms of error monitoring. J Exp Psychol: Gen 2000a;129:43-60.

Luu P, Flaisch T, Tucker DM. Medial frontal cortex in action monitoring. J Neurosci 2000b;20:464-9.

Luu P, Tucker DM, Derryberry D, Reed M, Poulsen C. Activity in human medial frontal cortex in emotional evaluation and error monitoring. Psychol Sci 2003;14:47-53.

Makeig S, Luu P, Tucker DM. Do 'brainstorms' help turn intentions into attentions? Paper Presented at the Human Brain Mapping, Sendai, Japan; 2002a.

Makeig S, Westerfield M, Jung T-P, Enghoff S, Townsend J, Courchesne E, et al. Dynamic brain sources of visual evoked responses. Science 2002b;295:690-4.

Makeig S, Debener S, Onton J, Delorme A. Mining event-related brain dynamics. Trends Cogn Sci 2004; in press.
Makeig S, Delorme A, Westerfield M, Jung T-P, Townsend J, Courchesne E, Sejnowski TJ. Electroencephalographic brain dynamics following manually responded visual targets. Submitted for publication

MEGIS, BESA, version 99, Munich, 99. 2000

Menon V, Adleman NE, White CD, Glover GH, Reiss AL. Error-related brain activation during a go/nogo response inhibition task. Hum Brain Mapp 2001;12:131-43.

Mizuki Y, Tanaka M, Isozaki H, Nishijima H, Inanaga K. Periodic appearance of theta rhythm in the frontal midline area during performance of a mental task. Electroencephalogr Clin Neurophysiol 1980;49(3/4):345-51.

Niki H, Watanabe M. Prefrontal and cingulate unit activity during timing behavior in the monkey. Brain Res 1979;171:213-24.

Perrin F, Pernier J, Bertrand O, Giard M, Echallier JF. Mapping of scalp potentials by surface spline interpolation. Electroencephalogr Clin Neurophysiol 1987;66:75-81.

Pickenhain L, Klingberg F. Hippocampal slow wave activity as a correlate of basic behavioral mechanisms in the rat. Prog Brain Res 1967;27: 218-27.

Powell DA, Joseph JA. Autonomic-somatic interaction and hippocampal theta activity. J Comp Physiol Psychol 1974;87:978-86.

Rüsseler J, Kuhlicke D, Münte TF. Human error monitoring during implicit and explicit learning of a sensorimotor response. Neurosci Res 2003;47: 233-40.

Sasaki K, Tsujimoto T, Nishikawa S, Nishitani N, Ishihara T. Frontal mental theta wave recorded simultaneously with magnetoencephalography and electroencephalography. Neurosci Res 1996;26(1):79-81.

Sayers BM, Beagley HA, Henshall WR. The mechanism of auditory evoked EEG responses. Nature 1974;247:481-3.

Scherg M, Berg P. New concepts of brain source imaging and localization. Electroencephalogr Clin Neurophysiol 1996;46:127-37.

Seidenbecher T, Laxmi TR, Stork O, Pape H-C. Amygdalar and hippocampal theta rhythm synchronization during fear memory retrieval. Science 2003;301:846-50.

Slobounov SM, Fukada K, Simon R, Rearick M, Ray W. Neurophysiological and behavioral indices of time pressure effects on visuomotor task performance. Cogn Brain Res 2000;9:287-98.

Tucker DM. Spatial sampling of head electrical fields: the geodesic sensor net. Electroencephalogr Clin Neurophysiol 1993;87:145-63.

Tucker DM, Luu P, Frishkoff G, Quiring J, Poulsen C. Frontolimbic response to negative feedback in clinical depression. $\mathrm{J}$ Abnorm Psychol 2003;112(4):667-78.

Ullsperger M, von Cramon DY. Subprocesses of performance monitoring: a dissociation of error processing and response competition revealed by event-related fMRI and ERPs. Neuroimage 2001;14:1387-401.

van Veen V, Carter CS. The timing of action monitoring processes in anterior cingulate cortex. J Cogn Neurosci 2002;14:593-602.

Vidal F, Hasbroucq T, Grapperon J, Bonnet M. Is the error negativity specific to errors? Biol Psychol 2000;51:109-28.

Vidal F, Burle B, Bonnet M, Grapperon J, Hasbroucq T. Error negativity on correct trials: a reexamination of available data. Biol Psychol 2003;64: 265-82. 